\title{
Inspiring Creative Constructivist Play
}

Chadwick A. Wingrave

$\mathrm{U}$ of Central Florida

Orlando, Florida 32816

cwingrav@eecs.ucf.edu

\section{Juliet Norton}

$\mathrm{U}$ of Central Florida

Orlando, Florida 32816

juliet@knights.ucf.edu

\section{Christopher Ross}

$\mathrm{U}$ of Central Florida

Orlando, Florida 32816

chillerman91@knights.ucf.edu

\section{Nathan Ochoa}

$\mathrm{U}$ of Central Florida

Orlando, Florida 32816

n_ochoa@knights.ucf.edu

\author{
Sergiu Veazanchin \\ $\mathrm{U}$ of Central Florida \\ Orlando, Florida 32816 \\ raiden@knights.ucf.edu
}

\section{Emiko Charbonneau}

$\mathrm{U}$ of Central Florida

Orlando, Florida 32816

miko@cs.ucf.edu

Joseph J. LaViola Jr.

$\mathrm{U}$ of Central Florida

Orlando, Florida 32816

jjl@eecs.ucf.edu

Copyright is held by the author/owner(s)

CHI'12, May 5-10, 2012, Austin, Texas, USA.

ACM 978-1-4503-1016-1/12/05.

\begin{abstract}
Inline with the BSCS 5E instructional model, we are using constructivist learning to teach physics in a heavily modified Minecraft game server. However, not all players like to be creative or are good at it. Because of this, we are creating three inspiration interventions to study their impact on learning and creative outcomes. Our model of inspiration incorporates three of the creativity resources defined by Investment Theory: knowledge, motivation and environment. We report on our current protocol, piloting of the protocol and our evolving methods of intervention creation.
\end{abstract}

\section{Author Keywords}

Creativity; Inspiration; Constructivist Learning; Physics

\section{ACM Classification Keywords}

K.3.0. Computers and Education: General

\section{Introduction}

We are exploring the intersection of creativity and learning, two separate processes, through constructivist play in the first person sandbox-building indie game Minecraft. Much of the games for education

phenomenon focuses on fun to motivate learning. The idea being that longer periods of exposure or repetition increase the amount of learning (e.g., the Math Blasters series). This "sugar coating" effect is often recognized by gamers for what it is: shallow gaming.

We take educational gaming one step further, by allowing the gamer to create new content for the game, 

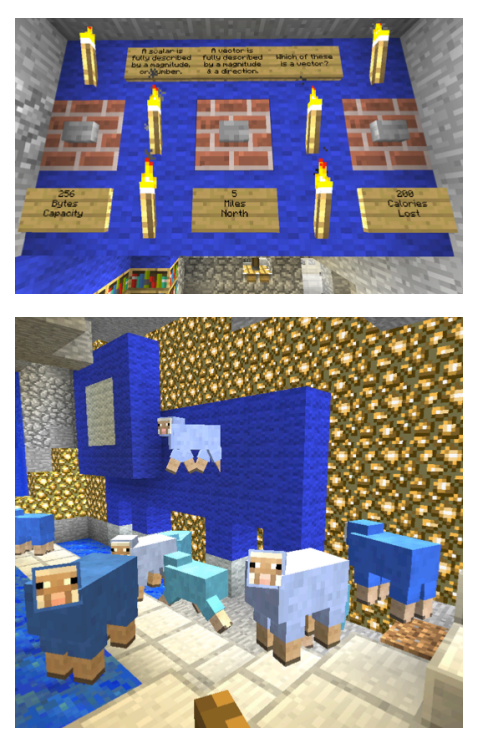

Figure 2. Not all players have knowledge of physics so we created in game instructional content for physics. Players must read and answer questions (top) that allow them to continue through a maze of rooms. We are also creating a story around the content, regarding hyperintelligent sheep (bottom), to entertain them as they navigate. Current development is extending this with plugins for interacting with physics phenomenon, such as kinematics, as they answer questions. a constructivist approach, and supporting their learning by scaffolding [8]. Scaffolding creates simpler learning representations, where mastery leads to knowledge applicable to a learning goal. Over time, the game fades the scaffolding in order to remain challenging, educational and motivating. In this way, we have a cycle of gaming to motivate learning and creating in a creative game to generate more gaming content (see Figure 1). But not all players will create, or are good at it, so we study inspiration.

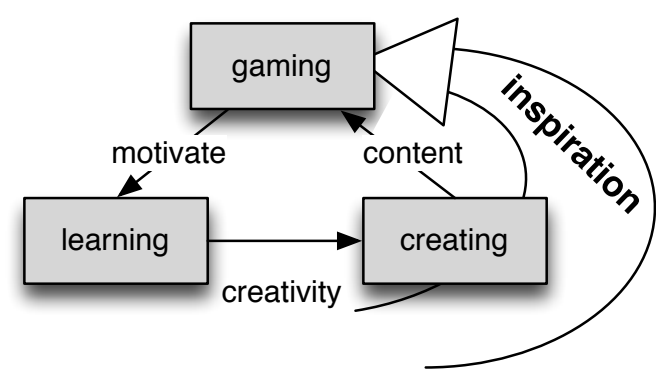

Figure 1. We are exploring a gaming, learning and creating cycle in constructivist learning. Players are motivated by the game to learn and through our inspiration interventions, to employ their creativity to make new content.

In this project, we are looking at how to inspire players to be creative in the development of educational physics themed quests. In one aspect, the game itself motivates, but inspiration is about feeding creativity: new approaches, new ideas, new learning experiences, different but complimentary to existing learning approaches. We are creating three inspirational interventions for players to see how they can use different aspects of Minecraft to tell a story, motivate play and create compelling places in the game world. This is in addition to the educational physics-related instruction we are developing in the world. The ultimate goal still remains learning outcomes, but we also take the stance that creativity is a learnable process [3] and measurable [5], and as such is an outcome itself.

\section{Background}

Minecraft

Minecraft ${ }^{1}$ is an open world style game that

paradoxically attracts the attention of mainstream gamers with: no plot, no story, no goal, simplistic combat and pixelated graphics [2]. Yet, it sold more than 3.5 million copies before it was even released and won the VGA 2011 indie game of the year. The game's main mechanic is creativity by placing, gathering and creating 1-meter cubes, but it also incorporates collaboration, exploration and adventure. It has grown to include tool crafting, survival mode, multiplayer, farming, livestock, Boolean logic and mechanical motion. As well, a developer community is actively modifying it through plugin creation.

In-Game Physics Instruction

We want players to design games based upon physics principles. Many popular games already use physics as their basic mechanic such as Portal or Angry Birds. However, not all players can understand game development or are experienced enough with physics to create such quests. So, we are creating in-game physics instruction to provide basic physics knowledge (see Figure 2). The instruction is designed to be fun, humorous and educational. The instruction originates from the online lessons at "The Physics Classroom"2 and provides the knowledge to players for integrating physics with their own creative ideas. We are also building plugins to extend Minecraft and assist end users in making quests out of physics. For example, one plugin creates regions that apply acceleration or velocity to players that enter or push a button.

Creativity and Inspiration

Creativity is "the ability to produce work that is both novel and appropriate" $[5, \mathrm{p} 3$.$] . Creative people add$

\footnotetext{
${ }^{1}$ http://minecraft.net

${ }^{2}$ http://physicsclassroom.com
} 
thoughts and ideas to a society, change it and drive it forward [4]. In a society unafraid of ideas, creative people are the cause of societal advancement. For this reason, creativity is a field of study important to, among others: science, art, business, mathematics and engineering. Creativity is usually not taught in schools $[5, \mathrm{p} 256]$ but it is a learned process that can be enhanced [5, chapter 20] and a game environment can provide positive reinforcement to encourage this type of thinking.

Creativity, according to Investment Theory [9], is built on six resources that fuel the creative person. It requires intelligence to judge ideas, knowledge of the domain, a thinking style that allows for novel thoughts, a personality that is willing to defy the norm,

motivation to overcome obstacles and an environment that reduces risk and obstacles and provides rewards.

Inspiration then, by our definition, is providing knowledge, motivation and an environment to be creative. Specifically, we inspire creativity by: 1 ) teaching domain knowledge in physics and skills to create quests, 2 ) motivating by example quests and fun educational content, 3) an environment that is easy to use, build and share creations for social rewards, 4) simply asking them to be creative [7].

\section{BSCS 5E Instructional Model}

The benefits of our approach, especially contrasted with traditional educational games, are demonstrated within the BSCS 5E instructional model [1]. This instructional model was created to help students learn fundamental concepts in science and other domains. We are using it in the construction of our physics education modules. It consists of five phases: engage - access prior

knowledge and promote curiosity; explore - use existing knowledge to generate new ideas, explore questions; explain - focus on experiences and demonstrate conceptual knowledge and introduce skills and deeper understanding; elaborate - challenge student understanding for broader and deeper understanding; evaluate - assess understanding and progress towards objectives. Where typical educational play involves engaging the player and giving them a game to explore some educational topic, constructivist play, where they create and build, requires students to reflect and deepen and broaden their understanding in the explain and elaborate phases.

\section{Learning and Creativity Intervention}

Our current focus is the development of an

experimental protocol where participants have the resources, instruction and guidance to create physics quests. Once the protocol is finalized, we will begin the piloting of the inspiration interventions and their effect on the learning and creative outcomes. Such a protocol is non-trivial as it requires training participants in how to play a game, in physics and in game development. At this time, we are avoiding actual implementation of this protocol in the game for our participant population as end-user development tools are being built $[13,11]$ In its place, we are using a verbal protocol to explain and refine the gameplay, in-sync with programming step-wise refinement [12], the first steps of ConceptOriented Design [11] and explain and elaboration phases of the BSCS $5 \mathrm{E}$ model [1].

Our protocol (at the time of this writing) begins with pre-tests in physics conceptual understanding [6] and then exposure to Minecraft so they learn about the environment they are expected to build a game in. We then ask them to describe a game idea that

incorporates velocity. We are also looking at the effect of giving the participants a theme to structure their thinking. In a final step, we ask them to stepwise describe the game with increasing levels of detail. At the end, we give them another physics test and a gameplay and demographic survey. A panel of judges evaluates the games.

We have iterated on the protocol to improve instruction to the participants and to make the process clearer. We have found that participants without physics knowledge or gaming experience tend to have problems. The participants also do not have a good understanding of 


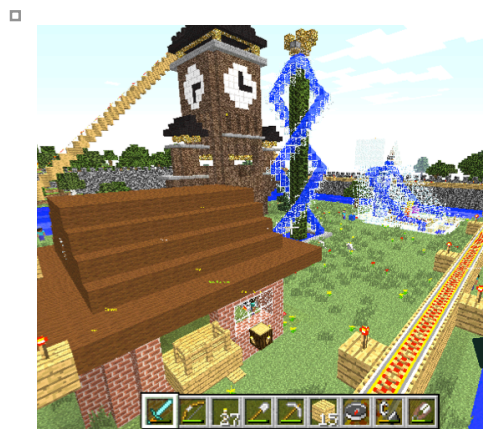

Figure 3. The Introduction World teaches how to play on our server but gives a compelling backstory using quests, NPCs and visual elements as well.

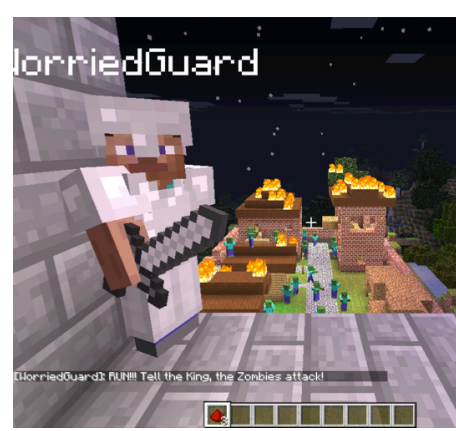

Figure 4. A view between puzzles shows zombies destroying a village which motivates the player while they learn Boolean logic. how to use physics in a game so we are working on physics quests as examples. We also feel that a longitudinal component of this vein of research is required and are working with freshmen physics instructors to incorporate our work into introductory physics classes. We are specifically targeting kinematics as they are commonly used in existing games.

\section{Inspiration Interventions (IIs)}

By exposing participants to inspiring examples of play inside the Minecraft game, we engage them and expect them to improve their motivation and knowledge of the game (two creativity resources according to Investment Theory [9]), which in turn drives their creativity and time spent learning. Our inspiration interventions are short instances (in terms of MMOGs) of play. They are iterated on in our group with constant feedback and we develop plugins and modifications to the Minecraft game as needed. Our play testers are from our own research group or part of our "power users" that play and build in our Minds of Chimera Minecraft server [10], coming in once a week to talk about their experiences.

\section{1: Introduction World}

While Minecraft has an in-game tutorial, we found it lacking, as many of our pilot users are not avid gamers. So we created a tutorial world that also serves to set the backstory of our server, Minds of Chimera (see Figure 3). In our server, the player's actions in the introduction world cause the benevolent Chimera deity to fracture into its separate animals, which are separate worlds for different play styles: lion for questing, snake for survival mode and goat for creative mode.

The introduction world started as a simple tutorial but after multiple iterations and incorporation of the server's backstory, it became one of our inspiration interventions. To create the feeling of impending doom, NPCs comment on things being fine, yet bridges are out, lava flows are appearing, dark walls surround the compound and fires are seen in the distance. Players complete a series of quests, to learn skills needed for our server, and it culminates in them entering a gatehouse to the outside world, which has failed. When the player pulls a lever, thinking it heals the world, they wake in Port City and begin to play on our server.

II 2: Zombie Attack

Zombie Attack follows a typical fantasy game plot line but utilizes Boolean logic programming as the gaming element to be mastered. It starts by waking up in a castle's barracks and guards telling you to begin your training. After a few rooms of normal training, learning basic Redstone wiring (Minecraft's in-game Boolean logic wiring system), the castle comes under attack from zombies (see Figure 4). The guards, busy defending the castle, charge you with running to the king to rally the troops but the castle's defenses must be bypassed. As the player uses Boolean logic to dismantle traps and open passages, the castle is crumbling around them and NPCs tell them to hurry. Each defense overcome moves the player one step closer to the king.

The original intent, before use as an inspiring environment, was to demonstrate Redstone wiring in Minecraft and develop instructional content on Boolean logic. In a typical instructional approach, we deconstructed the task of wiring into each bit of knowledge and developed a set of scaffolds to assist the player and a plan to remove the scaffolds as the player progressed. Once we could be sure that the educational material was comprehensible, we ran a series of pilot tests.

However, perhaps inspired ourselves, we saw ways to improve the lessons with story. Our first story was similar to the game Portal, where you must complete a series of rooms while harassed by an antagonist. This changed to a floating island; a fantasy setting that freed us from rooms and shifted us to teaching with puzzles. Motivating, but lacking a story, a plotline to defend the castle was used. The castle's puzzles were selected and ordered so that players would run through 


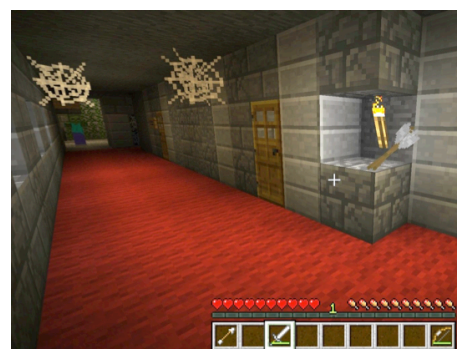

Figure 5. Even with simple blocks, we were able to create the desired feel. The hallways are dark (brightened for publication), cobweb filled and packed with zombies and skeletons. each room in a linear order with each puzzle requiring knowledge of a specific aspect of Redstone wiring. We also built sections around the puzzles, showing a town on fire, broken and burning walls, invading zombies and defending guard NPCs with desperate messages for the player. This was play tested and we reordered some puzzles. We also added the initial story-arc of training, as a means of scaffolding the most basic of Redstone wiring knowledge, so players have the basic requisite knowledge to complete the puzzles. Interesting elements of the gameplay:

- Repairing AND and OR gates were fun to play with when seen as powering doors and gates.

- Signs provide instruction, but break game presence. We created affordances to scaffold learning that kept them present in the game. As an example, blue wool showed a wire connecting to a power source and red wool showed the receiving end.

- We created effective NPC interaction that drove the player on, provided story and compelling scenes were shown, such as burning towns and zombie hordes fighting guards. This occurred despite Minecraft's pixelated look and feel.

- We began to experiment with sound and story. In the training camp, the player walks over a pressure plate that sets off hidden TNT to foreshadow events. A few feet up, they pass a startled NPC.

- Despite being surrounded by zombies at every turn, combat did not occur so player success is dictated by their puzzle solving ability. However, the zombie presence did keep players on edge and motivated.

\section{3: Haunted Mansion}

We explored the fear genre of gameplay in Minecraft, placing the player in the role of a butler in search of their employer, The Earl. Driven by a story-enriched objective, players venture forth encountering numerous puzzles and traps while fighting for their survival (see Figure 5 and Figure 6). The aspects we focused on were developing an "eerie atmosphere", invoking the fear of being lost, incorporating combat and rewarding curiosity, but creating risk with traps. There are three switches that the player must pull to open doors to the Earl, which at this point we find has transformed into the Zombie King and must be killed.

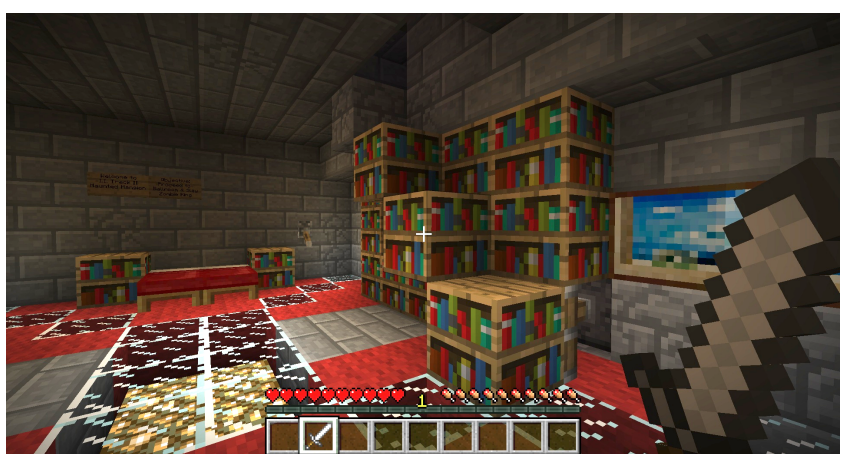

Figure 6. With mechanical blocks, we are able to create traps and secret areas. Here, the bedroom has a lever that turns the bookshelves into stairs to reach the roof.

The hallways and entry are filled with monsters that must be rushed through to get to the following rooms:

- A bedroom that is safe from monsters and has a hidden passage to an NPC to tell a backstory

- A library maze with cobwebs and a sliding shelf

- A dining room safe zone with a hole in the back wall, leading to a lava trapped chest

- A dilapidated green house with vines and some food growing, to help the player heal

- A creepy apothecary with cauldrons and devices shows the Earl's activities and whereabouts

- A basement maze of small, enclosed rooms with one-way doors and holes in the floor. The player 
quickly becomes lost until they realize the story is hinting at a pattern in the holes in the floor to guide them to the switch. When they come to a dead end they have to fall down to a dark labyrinth of cobwebs, monsters and water, before starting over in the rooms.

- A final encounter with the Earl, now Zombie King, in a large now destroyed ballroom

\section{Lessons Learned and Future Work}

The next steps are to finalize our protocol for physics quests creation and create the tools for end users to more readily add content. Both are under development and our end-user tool is informed by our own development of the inspiration interventions.

We have learned several things while creating quests and stories in Minecraft. First, though Minecraft has no innate quests and exists as a simple 1-meter block world, the number of blocks and ability to add plugins has resulted in a very expressive world. The lack of high-resolution graphics and built-in functionality is not missed. Second, Minecraft and its plugins have several methods of providing story, which is very useful to overcome game limitations, create a "feel" and train the player without having to give specific instructions. Third, we believe inspiration can play an interesting role in constructivist educational games. Our own skills as a group have grown regarding improved methods, plugin writing, puzzle creation, trap creation and story development. We expect the same for our upcoming participants and expect they will extend and build on what we are already doing. Fourth, our power users seem to have been inspired by our efforts, creating puzzles and traps on their own after even limited exposure, implying the inspiration is working.

\section{Acknowledgements}

We thank our pilots, power users and team members: Ross Arena, Monica Ramirez and Chris Zorn. This work is funded in part by NSF award \#IIS-0856045.

\section{References}

[1] Bybee, R. W., et al., "The BSCS 5E instructional model: Origins, effectiveness, and applications". (2006), BSCS: Colorado Springs, 49.

[2] Duncan, S. Minecraft, Beyond Construction and Survival.Well Played Journal, 1(1), 2011.

[3] Finke, R., Ward, T. \& Smith, S. Creative Cognition: Theory, Research and Applications. MIT Press, 1992.

[4] Florida, R. The Rise of the Creative Class and How It's Transforming Work, Leisure, Community and Everyday Life. New York: Basic Books, 2002.

[5] Handbook of Creativity edited by R. J. Sternberg, Cambridge University Press, 1999.

[6] Hestenes, D., Wells, M. \& Swackhamer, G., Force Concept Inventory. Physics Teacher, 30,141-158, 1992.

[7] Ironson, G. \& Davis, G. Faking High or Low Creativity Scores on the Adjective Check List. J. of Creative Behavior, 13, (1979), 139-145.

[8] Scaffolding student learning: Instructional approaches and issues. Hogan, K.(Ed);Pressley, M.(Ed), Brookline Books. (1997).

[9] Sternberg, R. An Investment Theory of Creativity and Its Development. Human Development, 34 (1991), 1-32.

[10] Wingrave, C. Minds of Chimera: Adapting Minecraft for a Creative Learning Platform, Learning Tech. Newsletter. (2011), 13(4).

[11] Wingrave, C., LaViola, J. and Bowman, D. (2009) A Natural, Tiered and Executable UIDL for 3D User Interfaces Based on Concept-Oriented Design." TOCHI, $16(4), 1-36$

[12] Wirth, N. 1983. "Program Development by Stepwise Refinement." Comm. of ACM 26(1): 70-74.

[13] Zorn, C., Wingrave, C. \& LaViola, J. Minecraft as a Platform for Increasing Interest in Programming through Child-like Block Play. Foundations of Digital Games, (submitted). 